\title{
1 Analgosedierung bei Adipositas
}

\author{
Christoph H.R. Wiese und Christoph L. Lassen
}

Die Analgosedierung adipöser Patienten stellt eine besondere Herausforderung in der Intensivtherapie dar. Einerseits ist zu gewährleisten, dass die Patienten schmerzfrei und von unangenehmen Reizen abgeschirmt sind und dass die unvermeidbaren intensivmedizinischen Maßnahmen erfolgen können, andererseits ist eine „Übersedierung“ zu vermeiden, da sie potenziell lebensbedrohliche Komplikationen wie langanhaltende maschinelle Beatmung, Gastroparese, $\mathrm{Hy}$ potension sowie Critical-Illness Polyneuro- und Myopathie hervorrufen kann. Insbesondere die Entwöhnung von der Beatmung bei nicht ausreichender Spontanatmung ist hier problematisch. In der Literatur gibt es kaum spezielle Betrachtungen der Analgosedierung bei adipösen Patienten. Daher folgt eine kurze Darstellung der aktuellen S3-Leitlinien zu Analgesie, Sedierung und Delirmanagement in der Intensivmedizin. In diesem Zusammenhang wird jeweils auf die spezifischen Besonderheiten der Analgosedierung bei adipösen Patienten eingegangen.

\subsection{S3-Leitlinien zur Analgesie, Sedierung und Delirmanagement}

Die S3-Leitlinien zu Analgesie, Sedierung und Delirmanagement fassen die aktuelle Datenlage zur Analgosedierung in der Intensivmedizin zusammen (DGAI u. DIVI 2010). Sie sind im Internet frei einsehbar und von Autoren verschiedenster Fachgesellschaften gemeinsam erstellt. Die eigentlichen Leitlinien für Erwachsene sind unterteilt in die Abschnitte:
- Monitoring,

- Therapie und Weaning,

- regionale Analgesieverfahren in der Intensivmedizin,

- neuromuskuläre Blockade in der Intensivmedizin,

- Ökonomie, Qualitätssicherung und Implementierung der Leitlinie zur Analgesie und Sedierung im Rahmen der Intensivmedizin und

- spezielle Patientengruppen in der Versorgung hinsichtlich Analgesie, Sedierung und Delir auf der Intensivstation.

Um auf die Besonderheiten im Rahmen der Analgosedierung bei adipösen Patienten eingehen zu können, erfolgt primär eine zusammenfassende Darstellung der ersten vier Abschnitte der oben zitierten S3-Leitlinie. Sekundär wird jeweils auf die Besonderheiten der Therapie bei Adipösen eingegangen.

\subsection{Monitoring}

\section{Analgesie}

Das Erleben von Schmerz stellt eine sehr belastende Erfahrung von Langzeitintensivpatienten dar. In diesem Zusammenhang ist vor allem das Selbstempfinden der Schmerzen zu erwähnen, das in deutlichem Widerspruch zur externen Schmerzeinschätzung der betreuenden Pflegekräfte und Ärzte aller an 
Tab. 1 Beispiele für validierte und allgemein eingesetzte Schmerzskalen

\begin{tabular}{|c|c|c|}
\hline Skala & Name & Kommentar \\
\hline NRS & Numerische Rating Skala & $\begin{array}{l}\text { eindimensionale Skala } \\
\text { Erhebung mittels Schmerzskalierung (Einteilung 0-10 = kein Schmerz bis stärkster } \\
\text { vorstellbarer Schmerz) } \\
\text { Selbsteinschätzung durch den Patienten }\end{array}$ \\
\hline VAS & Visuelle Analogskala & $\begin{array}{l}\text { eindimensionale Skala } \\
\text { Erhebung mittels Schmerzskalierung (Einteilung 0-100 = kein Schmerz bis stärkster } \\
\text { vorstellbarer Schmerz) } \\
\text { Selbsteinschätzung durch den Patienten }\end{array}$ \\
\hline VRS & Verbale Rating Skala & $\begin{array}{l}\text { eindimensionale Skala } \\
\text { Selbsteinschätzung nach Gruppen: } \\
\text { „keine“, „leichte“, „mittelstarke“, „starke“ und „sehr starke“ Schmerzen }\end{array}$ \\
\hline BPS & Behavioral Pain Scale & $\begin{array}{l}\text { Beobachtungsskala beim sedierten Patienten } \\
\text { Einteilung anhand Gesichtsausdruck, Bewegung der oberen Extremität und Adaptation an } \\
\text { das Beatmungsgerät } \\
\text { Bewertung jeweils mit 0-4 Punkten }\end{array}$ \\
\hline BESD & $\begin{array}{l}\text { Behandlung von } \\
\text { Schmerzen bei Demenz }\end{array}$ & $\begin{array}{l}\text { Beobachtungsskala beim dementen/deliranten Patienten } \\
\text { Einteilung anhand Atmung, Lautäußerung, Gesichtsausdruck, Körpersprache und Reaktion } \\
\text { auf Trost } \\
\text { Bewertung mit jeweils 0-2 Punkten }\end{array}$ \\
\hline
\end{tabular}

der Versorgung beteiligten Fachdisziplinen steht (Whipple et al. 1995).

Die Schmerzstärke sollte auch bei Intensivpatienten objektiv darstellbar sein. Hierfür ist die regelhafte Erhebung und Dokumentation von Schmerzwerten mithilfe validierter Skalen sowohl für wache als auch sedierte Patienten im Verlauf der Therapie unverzichtbar (s. Tab. 1). Zusätzlich ist eine individuelle Interventionsgrenze, einerseits durch die Therapeuten, andererseits aber auch patientenindividuell festzulegen (individuelles Empfinden von Schmerz ist nur bedingt durch allgemein gültige standardisierte Skalenwerte $z u$ verifizieren), ab der eine analgetische Therapie erfolgt, z.B. Numerische Rating Skala $(\mathrm{NRS})>3$.

\subsection{Sedierung}

Zum Monitoring der Sedierung sollen ebenso wie zum Analgesiemonitoring validierte Skalen eingesetzt werden. Dabei wird ein individuelles Sedierungsziel vorgegeben, das regelmäßig überprüft werden muss. Den Goldstandard stellt in diesem Zu- sammenhang die validierte Richmond-Agitation-Sedation-Scale (RASS) dar (Ely et al. 2003). Andere mögliche Skalen zur Einschätzung der Sedierungstiefe sind z.B. die Ramsay-Sedation-Scale (RSS) oder die Sedation Agitation Scale (SAS).

Unterstützend zur Skalenerhebung können auch apparative Verfahren zum Einsatz kommen. Gerade bei tiefer Sedierung und/oder neuromuskulärer Blockade können diese dazu beitragen, eine gewünschte Sedierungstiefe zu erreichen. Mögliche Verfahren sind u.a. EEG, Bispectraler Index (BIS) und akustisch evozierte Potenziale (AEP). Keines dieser Verfahren ist jedoch in der breiten Anwendung für Intensivpatienten validiert, insbesondere ist $u$.a. der Einfluss anderer im therapeutischen Verlauf auf der Intensivstation häufig eintretender Faktoren (z.B. Stoffwechselentgleisungen) auf die Messergebnisse nicht abschließend validiert und geklärt.

\section{Zur Situation bei adipösen Patienten}

Die vorgestellten Skalen finden in dieser Form auch bei adipösen Patienten Anwendung. Um eine be- 
darfsadaptierte Sedierung insbesondere bei dieser Patientengruppe zu gewährleisten, wird empfohlen, diese Skalen häufiger als in den Leitlinien angegeben $\mathrm{zu}$ erheben (z.B. alle 4 Stunden, also $2 \mathrm{x}$ pro Schicht). Insbesondere zur Vermeidung möglicher erhöhter Sedierung und damit einer insgesamt schlechteren Gesamtsituation im Rahmen der Langzeitsedierung sollten zusätzlich apparative Verfahren genutzt werden, um so zwischen möglicher Schmerzreaktion und Sedierung des Patienten unterscheiden zu können. Hierbei stehen z.B. mit dem BIS-Monitoring mehrere vergleichsweise einfach zu handhabende Geräte zur Verfügung. Somit lassen sich eine objektivierbare Sedierungstiefe und mögliche Schmerzen erfassen.

\subsection{Therapie und Weaning}

\section{Analgesie}

Je nach geplanter/voraussichtlicher Dauer der Analgosedierung werden unterschiedliche Substanzen zum Einsatz empfohlen. Eine zentrale Rolle nehmen dabei die Opioide ein. Eine Übersicht hierzu gibt Tabelle 2 .

Als Nicht-Opioid-Analgetikum empfiehlt sich in der Therapie Metamizol, da es problemlos kontinuierlich intravenös (z.B. $5 \mathrm{~g} / 24 \mathrm{~h}$ ) appliziert werden kann. Als Dauerinfusion reagieren die wenigsten Patienten mit kreislaufrelevanten Nebenwirkungen auf Metamizol. Dieses kann sich bei Kurzinfusionen alle $6 \mathrm{~h}$

Tab. 2 Medikamente zur Analgesie (Angaben entsprechend nach S3-Leitlinie)

Basisanalgesie (unabhängig von der Analgosedierungsdauer) Nicht-Opioid Analgetika

Regionalanästhesie

Analgosedierung $<72$ Stunden

Piritramid (Boli oder Patienten-kontrollierte Analgesie [PCA]) Sufentanil

Remifentanil

Analgosedierung $>72$ Stunden

Sufentanil

Fentanyl

Adjuvante Medikation

Clonidin

Ketamin (nur zusammen mit Benzodiazepinen oder Propofol) anders darstellen. Nicht-steroidale Antirheumatika (NSAR) und Coxibe bieten sich eher nicht an aufgrund der größeren Gefahr einer Nephrotoxizität in der intensivmedizinischen Behandlungssituation. Paracetamol ist analgetisch nicht so potent wie Metamizol und daher nur als Medikament zweiter Wahl zu bezeichnen.

Für die kurzfristige Analgosedierung und schmerztherapeutische Versorgung kann Piritramid bzw. Oxycodon inject nach Bedarf als Bolus oder in regelhaften Abständen gegeben werden. Beim nur leicht sedierten Patienten kann theoretisch auch eine PCA-Pumpe zum Einsatz kommen, durch die das Schmerzverhalten des Patienten insgesamt gut beurteilt werden kann. Außerdem empfiehlt sich der Einsatz von kurzwirksamen und relativ gut steuerbaren Opioiden. Von der Steuerbarkeit ist hier das Remifentanil wegen der kurzen und nicht-kontextsensitiven Halbwertszeit den anderen Opioiden deutlich überlegen. Gegen Remifentanil sprechen der hohe Preis und der abrupte Analgesieverlust nach Ausstellen des Perfusors.

Für die langfristige Analgosedierung können Sufentanil und Fentanyl empfohlen werden. Hier scheint Sufentanil Vorteile zu besitzen, u.a. wegen geringerer Kumulation und geringerer Toleranzentwicklung.

In der Weaningphase, auch nach Langzeit-Analgosedierung, sollte wieder ein kurzwirksames Opioid zum Einsatz kommen, bzw. eine Bolusgabe vorgenommen werden. Wenn hier Remifentanil benutzt wird, muss darauf geachtet werden, dass nach Beendigung der Remifentanilgabe ein langwirksames Opioid (z.B. Piritramid) verabreicht wird. Ansonsten kann es zu einem Opioid-Entzugssyndrom kommen.

Zur Einsparung von Analgetika können Clonidin und/oder Ketamin eingesetzt werden. Bei der Gabe von Ketamin ist auf eine entsprechende Sedierung mit Benzodiazepinen oder Propofol zu achten, um die unerwünschten psychomimetischen Nebenwirkungen von Ketamin zu vermeiden.

\section{Zur Situation bei Adipösen}

Die Empfehlungen der Leitlinien gelten auch für adipöse Patienten. Insbesondere diese sind jedoch durch eine Überdosierung des Opioids gefährdet. Die Gefahr der Akkumulation von Opioiden ist gerade bei kontinuierlicher Applikation aufgrund der hohen Lipophilie von Fentanyl und Sufentanil gegeben. Auch wenn es hierzu keine Daten in der Literatur gibt, empfehlen wir die frühzeitige Anwendung von Remifentanil, auch in der Langzeitsedierung. 
Im Weaning stellt Remifentanil ein quasi ideales Opioid dar. Es kann ein hohes Analgesieniveau ohne die Gefahr der Akkumulation aufrechterhalten werden.

Zur Extubation nach erfolgreichem Spontanatemversuch kann entweder die kontinuierliche Remifentanilapplikation durch titrierte Bolusgaben ersetzt werden. Es besteht aber auch die Möglichkeit, die Remifentanilinfusion auf niedriger Stufe weiterzuführen und die Dosis nach Extubation weiter langsam zu reduzieren.

Wegen der hohen Lipophilie der Opioide besteht bei Adipösen - insbesondere bei Dauerinfusion - die Gefahr der Überdosierung.

Insgesamt kann bei adipösen Patienten keine Dosisempfehlung der Opioide bezogen auf das Körpergewicht gegeben werden. Die Dosierung sollte sich strikt an dem klinischen Bedarf (Schmerzskala/Sedierungsgrad) orientieren. Der Einsatz von adjuvanten Medikamenten ist bei adipösen Patienten sinnvoll, um eine zu hohe Dosierung der Opioide zu vermeiden. In diesem Zusammenhang gibt es auch keine spezifischen Anpassungen an das Körpergewicht, sondern einen zielgerichteten, an den Symptomen und der individuellen Verträglichkeit orientierten Versuch, ein günstiges Wirkungs-Nebenwirkungsprofil zu erreichen.

\section{Sedierung}

Die tiefe Sedierung ist nur speziellen Indikationen vorbehalten. Anzustreben ist eine Sedierung, die, wenn notwendig, die maschinelle Beatmung ermöglicht, und zu einem ruhigen nicht agitierten Patienten führt. Die Sedierung sollte unter Festlegung eines Sedierungszieles anhand eines Protokolls erfolgen. Wenn möglich, sollten Sedierungspausen mit kurzen Wachheitsphasen durchgeführt werden (Girard et al. 2008).

Die Auswahl der zur Sedierung benutzten Medikamente richtet sich nach der voraussichtlichen Sedierungsdauer:

- Beikurzer Sedierung < 7 Tage wird der kontinuierliche Einsatz von Propofol oder die Bolusapplikation von Midazolam empfohlen. Alternativ kann der Einsatz von Narkosegasen mittels spezieller Applikatorsysteme $\left(\right.$ AnaConDa $\left.^{\circledR}\right)$ erwogen werden.

- Bei längerer Sedierung wird Propofol wegen der Gefahr des Propofol-Infusionssyndroms und der fehlenden Überlegenheit zu Midazolam nicht empfohlen (Walder et al. 2001). Stattdessen kommen Midazolam oder Lorazepam als Bolus zum Einsatz.

Wie bei der Analgesie können auch bei der Sedierung Clonidin und/oder niedrig dosiertes Ketamin die Dosis der Sedativa reduzieren.

\section{Zur Situation bei Adipösen}

Analog zur Therapie mit Analgetika gilt auch für die Sedativa, dass die Dosierung der Substanzen nicht nach Körpergewicht sondern nach Bedarf erfolgt. Der Bedarf kann anhand der Sedierungsskalen abgeschätzt werden. Gerade für adipöse Patienten wird empfohlen, dass zusätzlich zu den Skalen die Sedierung apparativ unterstützt erfasst wird. Hierdurch kann eine „Übersedierung“ im Vergleich zu rein gewichtsdosierter Sedierung verhindert werden ( $\mathrm{La}$ Colla et al. 2009).

\section{Besonders bei Adipösen besteht die Gefahr der Kumu- lation von Benzodiazepinen, z.B. Midazolam, im Fett- gewebe. Von einer Dauerinfusion solcher Substan- zen mittels Perfusor ist bei dieser Patientengruppe dringend abzuraten.}

Alternativ zur intravenösen Sedierung kann auch eine inhalative Sedierung durchgeführt werden. Hierzu liegen jedoch keine Studien bei adipösen Patienten vor.

Der Einsatz von Clonidin und Ketamin ist auch zur adjuvanten Sedierung adipöser Patienten sinnvoll, um eine Dosismaximierung eines Monotherapeutikums zu vermeiden.

\subsection{Regionale Analgesieverfahren in der Intensivmedizin}

Unter strenger Beachtung der Kontraindikationen kann in Einzelfällen nach Risiko-Nutzen-Abwägung eine Regionalanästhesie auch bei beatmeten Intensivpatienten eingesetzt werden. Hierbei kommt hauptsächlich die Epiduralanalgesie zum Einsatz. Da meist ein ausreichend hohes Analgesieniveau bis zum Oberbauch erreicht werden soll und damit auch eine Therapie einer Darmparalyse möglich ist, ist prinzipiell ein thorakaler Epiduralkatheter zu erwägen. Zur Therapiesicherheit sollte der neurologi- 
sche Status nach Anlage, z.B. im Rahmen von täglichen Sedierungspausen, erhoben werden. Der Einsatz erscheint insbesondere im Rahmen des Weaning sinnvoll, um eine Reduktion der Opioiddosis zu erreichen.

\section{Zur Situation bei Adipösen}

Die Anlage einer thorakalen Epiduralanalgesie beim adipösen Intensivpatienten stellt eine hohe technische Herausforderung dar, daher sollte sie nur durch einen in dem Verfahren geübten Anästhesisten erfolgen. Bei nicht einwilligungsfähigen Patienten ist die Risikoaufklärung und Einwilligung über einen Betreuer einzuholen und zu dokumentieren. Auf eine atraumatische Punktion ist $\mathrm{zu}$ achten, ggf. muss die Anlage abgebrochen werden. Bei erfolgreicher Anlage kann die thorakale Epiduralanalgesie insbesondere im Weaning adipöser Patienten einen wertvollen Therapiebeitrag leisten, da es hierdurch zu einer relevanten Einsparung von Opioiden kommen kann.

\subsection{Neuromuskuläre Blockade in der Intensivmedizin}

Medikamente zur neuromuskulären Blockade in der Intensivmedizin werden zunehmend restriktiv eingesetzt. Die Nebenwirkungen einer kontinuierlichen neuromuskulären Blockade sind zu bedenken; diese sind unter anderem erhöhte Pneumonieraten, Atelektasenbildung und prolongierte Muskelschwäche mit der Unmöglichkeit der Entwöhnung von der Beatmung. Sollten Medikamente zur neuromuskulären Blockade eingesetzt werden, ist ein neuromuskuläres Monitoring obligat (z.B. Train-of-four, TOF).

\section{Zur Situation bei Adipösen}

Man kann davon ausgehen, dass Patienten mit Adipositas eine geringere Toleranz der möglichen Nebenwirkungen (z.B. pulmonale Komplikationen, Muskelschwäche) haben. Daher sollte der Einsatz von Medikamenten zur neuromuskulären Blockade nur im Einzelfall erfolgen.

\section{Fazit für die Praxis}

Bei adipösen Patienten sollte die Analgosedierung den Empfehlungen der S3-Leitlinie folgen. Zu achten ist auf eine bedarfsadaptierte Dosierung der Medikamente zur Analgosedierung. Die Dosierung richtet sich nach den erhobenen Schmerz- und Sedierungsskalen. Eine apparative Unterstützung der Bestimmung der Sedierungstiefe erscheint sinnvoll. Es sollten Medikamente mit kurzer Halbwertszeit und geringer Kumulationstendenz bevorzugt werden, z.B. Propofol/Remifentanil unterstützt durch den Einsatz von adjuvanter Medikation. Darüber hinaus ist im Einzelfall zu prüfen, ob die kontinuierliche Zufuhr analgosedierender Substanzen wegen der hohen Kumulationsgefahr (Lipophilie!) besser durch intermittierende Bolusgaben ersetzt wird. Eine neuromuskuläre Blockade erscheint wegen der möglichen Nebenwirkungen nur in wenigen Fällen sinnvoll.

\section{Literatur}

Deutsche Gesellschaft für Anästhesiologie und Intensivmedizin (DGAI), Deutsche Interdisziplinäre Vereinigung für Intensiv- und Notfallmedizin (DIVI) (Hrsg.) (2010) Analgesie, Sedierung und Delirmanagement in der Intensivmedizin. http://www.awmf. org/leitlinien/detail/Il/001-012.html, abgerufen am 17.11.2011

Ely EW, Truman B, Shintani A, Thomason JW, Wheeler AP, Gordon S, Francis |, Speroff T, Gautam S, Margolin R, Sessler CN, Dittus RS, Bernard GR (2003) Monitoring sedation status over time in ICU patients: reliability and validity of the Richmond Agitation-Sedation Scale (RASS). JAMA 289, 2983-2991

Girard TD, Kress JP, Fuchs BD, Thomason JW, Schweickert WD, Pun BT, Taichman DB, Dunn JG, Pohlman AS, Kinniry PA, Jackson JC, Canonico AE, Light RW, Shintani AK, Thompson JL, Gordon SM, Hall JB, Dittus RS, Bernard GR, Ely EW (2008) Efficacy and safety of a paired sedation and ventilator weaning protocol for mechanically ventilated patients in intensive care (Awakening and Breathing Controlled trial): a randomised controlled trial. Lancet 371, 126-134

La Colla L, Albertin A, La Colla G, Ceriani V, Lodi T, Porta A, Aldegheri G, Mangano A, Khairallah I, Fermo I (2009) No adjustment vs. adjustment formula as input weight for propofol target-controlled infusion in morbidly obese patients. Eur | Anaesthesiol 26, 362-369

Walder B, Elia N, Henzi I, Romand JR, Tramer MR (2001) A lack of evidence of superiority of propofol versus midazolam for sedation in mechanically ventilated critically ill patients: a qualitative and quantitative systematic review. Anesth Analg 92, 975983

Whipple JK, Lewis KS, Quebbeman E), Wolff M, Gottlieb MS, Medicus-Bringa M, Hartnett KR, Graf M, Ausman RK (1995) Analysis of pain management in critically ill patients. Pharmacotherapy $15,592-599$ 


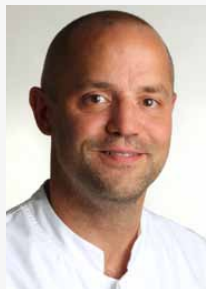

\section{PD Dr. med. Christoph H.R. Wiese}

Nach dem Medizinstudium an der Georg-Augustin-Universität Göttingen Beginn der klinischen Tätigkeit 1999 am Klinikum Braunschweig in der Klinik für Anästhesiologie. Von 20022008 Arzt in Weiterbildung am Uniklinikum Göttingen. 2002 Promotion. Ab 2004 Facharzt für Anästhesiologie und Erlangung der Zusatzbezeichnungen Notfallmedizin, Intensivmedizin, spezielle Schmerztherapie und Palliativmedizin. Seit 2009 Oberarzt der Klinik für Anästhesiologie am Uniklinikum Regensburg sowie Leiter der interdisziplinären Schmerzambulanz. 2010 Habilitation zum Thema „Palliative Notfälle - eine neue Herausforderung für die Notfallmedizin“.

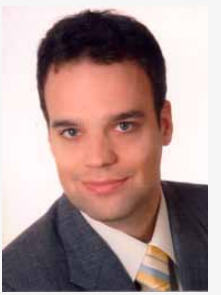

\section{Dr. med. Christoph L. Lassen}

Nach dem Medizinstudium an der Christian-Albrechts-Universität Kiel mit Forschungsaufenthalt an der University of Utah in Salt Lake City, USA, arbeitete er seit 2005 als Arzt in Weiterbildung am Universitätsklinikum Regensburg in der Klinik für Anästhesiologie. 2009 Promotion. Besondere Interessensschwerpunkte sind Schmerztherapie und Palliativmedizin. 